\title{
Factores biopsicosociales asociados a la disfunción sexual femenina en una población mexicana
}

\author{
Genaro Vega M. ${ }^{1}$, Patricia Jezabel Flores C. ${ }^{1}$, Javier Ávila M. ${ }^{1}$, Alma Becerril S. ${ }^{1}$, \\ Alfredo Jesús Vega M. ${ }^{1}$, Nicolás Camacho C. ${ }^{1}$, Pablo García S. ${ }^{1}$ \\ ${ }^{1}$ Facultad de Medicina, Universidad Autónoma de Querétaro, México.
}

\section{RESUMEN}

Objetivo: Determinar los factores biopsicosociales asociados a la disfunción sexual femenina en una población mexicana. Métodos: Estudio comparativo en mujeres que acudieron como acompañantes a una Unidad de Medicina Familiar de Querétaro, México. Se aplicaron las encuestas de Laumman, Chávez y Velazco, Faces III, y Grajales para valorar sexualidad, funcionalidad conyugal, funcionalidad familiar y estado de la autoestima. Los resultados fueron analizados con Odds Ratios, Chi cuadrado y t de Student. Resultados: De 110 mujeres entrevistadas, 65 (59\%) refirieron alguna disfunción sexual y 45 (41\%) lo negaron. Se formaron aleatoriamente dos grupos de 44 mujeres: con y sin disfunción sexual. Los factores que se asociaron significativamente a las alteraciones sexuales fueron: edad mayor a 40 años, laborar fuera del hogar, presencia de obesidad y/o sobrepeso, hipertensión arterial, diabetes mellitus, histerectomía previa, maternidad, disfuncionalidad conyugal, autoestima alta, depresión leve a severa, familia disfuncional, estrato socioeconómico bajo. Las alteraciones sexuales más frecuentes fueron: disminución en el deseo sexual $(34,1 \%)$, disfunción eréctil de la pareja $(22,7 \%)$, dispareunia y falta de excitación $(20,5 \%)$, sexo referido como desagradable e incapacidad para llegar al orgasmo $(13,6 \%)$, ansiedad por el desempeño sexual $(6,8 \%)$ y eyaculación precoz $(4,5 \%)$. Conclusiones: Es importante identificar los factores que afectan la sexualidad femenina para ofrecer un manejo multidisciplinario y prevenir implicaciones a nivel del entorno familiar y conyugal.

\section{PALABRAS CLAVE: Disfunción sexual femenina, factores biopsicosociales}

\section{SUMMARY}

Objective: To determine the biopsychosocial factors associated with female sexual dysfunction in a Mexican population. Methods: A comparative study in women who were attended at a Family Medicine Unit of Queretaro, Mexico. Surveys of Laumman, Chavez and Velazco, Faces III, and Grajales, were applied to assess sexuality, marital functionality, familiar functionality and state self-esteem. The results were analyzed with Odds Ratios, chi-square and Student t test. Results: Of 110 women interviewed, 65 (59\%) reported sexual dysfunction and $45(41 \%)$ denied. They formed two groups randomly from 44 women with and without sexual dysfunction. Factors that were significantly associated with sexual disorders were: age greater than 40 years, labor outside the home, presence of obesity and / or overweight, hypertension, diabetes mellitus, prior hysterectomy, maternity, marital dysfunction, high self-esteem, mild to severe depression, dysfunctional family, low socioeconomic status. The most common sexual dysfunction were: decrease in sexual desire (34.1\%), erectile dysfunction couples $(22.7 \%)$, dyspareunia and lack of arousal 
$(20.5 \%)$, referred to as unpleasant sex and inability to reach orgasm $13.6 \%$ ) sexual performance anxiety $(6.8 \%)$ and premature ejaculation (4.5\%). Conclusions: It is important to identify the factors affecting female sexuality to offer multidisciplinary management and prevention implications at the level of family and marital environment.

\section{KEY WORDS: Female sexual dysfunction, biopsychosocial factors}

\section{INTRODUCCIÓN}

Para el siglo XXI la expectativa de vida en la mujer ha aumentado por sobre los 75 años, su estilo de vida se ha modificado, haciéndose más activa física, intelectual y sexualmente (1-3).

El término de sexualidad representa una interacción de valores y prácticas socioculturales compleja, la actividad sexual comprende los aspectos biológicos y sociales de los individuos $(4,5)$. Involucra la percepción y control corporal, el juego de la satisfacción placentera y rechazo, como también las limitantes culturales y religiosas (6-7).

Existen diversos factores biológicos, sicológicos, sociales, farmacológicos, ambientales y de salud, que intervienen o pueden alterar la función sexual ocasionando alguna manifestación en la relación sexual de pareja (8-13). En ocasiones estas alteraciones de la sexualidad pueden derivar en problemas serios en la relación matrimonial y familiar (14).

Existen varias clasificaciones que auxilian la delimitación de la diversidad de disfuncionalidad sexual en ambos sexos, de las más recientes es por parte de la Asociación Psiquiátrica Americana en el 2002 las cuales mencionan los siguientes rubros: trastornos del deseo sexual, trastornos de la excitación sexual, trastornos del orgasmo, trastornos sexuales dolorosos, disfunción sexual secundaria a condición médica general, disfunción sexual debida a substancias, así como la clasificación del DSM IV: trastornos del deseo sexual, trastornos de la respuesta sexual y trastornos dolorosos (15-17).

Evidentemente la evaluación de la mujer que presente alteraciones sexuales es multidisciplinaria para identificar los factores biopsicosociales que puedan provocar una disfunción para lograr un diagnóstico y tratamiento eficaz (18-19).

El objetivo de este estudio es determinar los factores biopsicosociales asociados a la disfunción sexual femenina en una población mexicana.

\section{PACIENTES Y MÉTODO}

Se realizó un estudio epidemiológico de casos $y$ controles efectuados en mujeres que acudieron como acompañantes de pacientes en la consulta externa de una unidad de Medicina Familiar en la ciudad de Querétaro, México, en el período de enero a diciembre de 2011. Previo consentimiento informado se les aplicó la encuesta de Laumman y cols (20), para estudiar la presencia de alteraciones sexuales. Se formaron dos grupos seleccionados aleatoriamente de una muestra de 110 mujeres encuestadas: Grupo 1 formado por 44 mujeres con alguna disfunción sexual, y Grupo 2 formado por 44 mujeres sin disfunción sexual

A ambos grupos se les aplicaron las siguientes encuestas ya validadas por estudios previos en México: el cuestionario de Chávez y Velazco (21) para medir la funcionalidad conyugal, el cuestionario de Grajales y Valderrama (22) para medir el estado de autoestima. La depresión se evaluó con la escala de Yesavage (23), la funcionalidad familiar se midió con la prueba de Apgar familiar (24), finalmente para el estado socioeconómico se aplicó el método de Graffar (25).

Se estudiaron además las siguientes variables: socio demográficas (edad, ocupación, lugar de residencia, estado civil, estado socioeconómico), reproductivas (número de gestaciones), nutricionales (sobre peso y obesidad) toxicomanías (tabaquismo, alcoholismo, tabaquismo asociado al alcoholismo), antecedentes quirúrgicos (histerectomía previa), patologías asociadas (hipertensión arterial, diabetes mellitus, cardiopatías, etc.), y tipo de alteración sexual.

Para el análisis se utilizó el programa SPSS V-17, aplicando estadística descriptiva con moda y porcentajes, y estadística inferencial con la prueba de chi cuadrada y Odds Ratio para medir la fuerza de asociación, con un intervalo de confianza del 95\% (IC95\%). Se consideró diferencia significativa un valor $p<0,05$.

El protocolo de investigación fue evaluado y aprobado por el comité de investigación de la Facultad de Medicina de la Universidad Autónoma de Querétaro, México. Se contó con el consentimiento informado de las mujeres encuestadas y con la garantía de confidencialidad y veracidad de los resultados. 


\section{RESULTADOS}

Se entrevistaron a 110 mujeres con vida sexual activa de las cuales $65(59 \%)$ refirieron al menos alguna disfunción sexual, mientras que $45(41 \%)$ lo negaron. Se formaron dos grupos seleccionados al azar de la muestra anterior: Grupo 1 formado por 44 mujeres con alguna disfunción sexual, y Grupo 2 formado por 44 mujeres sin disfunción sexual.

De los factores analizados que presentaron diferencia estadísticamente significativa fueron (Tabla I): edad mayor a 40 años, trabajo fuera del hogar, obesidad y/o sobrepeso, hipertensión arterial, diabetes mellitus, histerectomía previa, maternidad, disfuncionalidad conyugal, autoestima alta, depresión leve a severa, familia disfuncional, estrato socioeconómico bajo. No mostraron ser factores de riesgo: estado civil, pareja estable, lugar de residencia, escolaridad, número de gestas, ejercicio, tabaquismo, alcoholismo, tabaquismo asociado al alcoholismo, enfermedades concomitantes como cardiopatías, artritis, colitis, tratamiento hormonal.

Las alteraciones sexuales más frecuentes fueron (Tabla II): disminución del deseo sexual $(34,1 \%)$, disfunción eréctil de la pareja $(22,7 \%)$, dispareunia y falta de excitación $(20,5 \%)$, sexo referido como desagradable e incapacidad para llegar al orgasmo $(13,6 \%)$, ansiedad por el desempeño sexual $(6,8 \%)$ y eyaculación precoz $(4,5 \%)$.

\section{DISCUSIÓN}

El modelo de respuesta sexual entre el sexo femenino y masculino debe de ser motivo de una individualización del análisis de las alteraciones sexuales que presenta el paciente. Un ejemplo de variantes entre géneros se puede observar en el estudio de Kammerer y Roger (17), que en el 30\%

Tabla I

FACTORES ASOCIADOS A LA DISFUNCIÓN SEXUAL FEMENINA

\begin{tabular}{lccc}
\hline Factores asociados & Valor $\mathrm{p}$ & OR & IC95\% \\
\hline Edad mayor de 40 años & 0,000 & 26,8 & $13,04-217,43$ \\
Síndrome climatérico & 0,000 & 21,0 & $4,33-101,67$ \\
Diabetes mellitus & 0,011 & 13,5 & $1,55-117,13$ \\
Laborar fuera del hogar & 0,014 & 12,5 & $1,43-108,27$ \\
Antecedentes histerectomía & 0,001 & 12,4 & $2,41-64,49$ \\
Bajo nivel socioeconómico & 0,005 & 1,94 & $1,88-33,49$ \\
Paridad $\geq 3$ partos & 0,012 & 6,76 & $1,60-28,54$ \\
Disfuncionalidad conyugal & 0,012 & 6,22 & $1,56-24,71$ \\
Obesidad/Sobrepeso & 0,01 & 5,46 & $1,62-18,35$ \\
Familia disfuncional & 0,022 & 4,57 & $1,38-15,11$ \\
\hline
\end{tabular}

Tabla II

\section{ALTERACIONES SEXUALES MÁS FRECUENTES}

\begin{tabular}{lc}
\hline Disfunción sexual & $\%$ \\
\hline Disminución en el deseo sexual & 34,1 \\
Disfunción Eréctil de la pareja & 22,7 \\
Dispareunia y falta de excitación & 20,5 \\
Sexo desagradable e incapacidad & 13,6 \\
para llegar al orgasmo & \\
Ansiedad por el desempeño sexual & 6,8 \\
Eyaculación precoz & 4,5 \\
\hline
\end{tabular}

de las mujeres refieren tener un orgasmo durante su actividad sexual rutinaria, en contra del $75 \%$ de los hombres entrevistados. En nuestro estudio la mayoría de las encuestadas respondieron afirmativamente a la presentación de orgasmo y solo una pequeña parte entendió a la relación sexual como desagradable o sin tener orgasmo.

En un estudio realizado por Laumman y cols (20), se encontró que el $43 \%$ de la población general tenía alguna alteración sexual y la entidad patológica más relacionada en ambos sexos fue la enfermedad arterial periférica. 
En otro estudio realizado por McCall-Hosenfeld y cols (26), encontraron que cuando la alteración sexual en la mujer está asociada a alguna enfermedad crónica degenerativa, la recomendación de cambios en el estilo de vida y de los hábitos higiénicos y dietéticos, mejoran sus condiciones vasculares y metabólicas que repercute en una mejor vida sexual.

En nuestro estudio el aspecto emocional y psicológico no tuvo variantes en alguno de los sexos. De los factores asociados llama la atención por su frecuencia la edad y tener un trabajo fuera del hogar. La edad pudiera estar explicada por los cambios hormonales propios de la pre menopausia y climaterio, que ocasionan en una gran parte de la población femenina una disminución del deseo sexual, y por ende poca excitación, dispareunia y falta de orgasmo, por lo que refieren al sexo como desagradable.

El laborar fuera del hogar, fue el factor asociado a la disfunción femenina más frecuente después de la edad, probablemente el que la mujer juegue un doble rol en la dinámica familiar, ama de casa y trabajadora, provoque un cansancio físico y emocional que redunda en la calidad de la relación conyugal, similar a lo reportado por Clayton y cols (18), quienes en su estudio el $24 \%$ de las mujeres presentaron algún tipo de problema conyugal, y laborar fuera del hogar pudiera participar como único factor o bien como detonante, cuando yacen entidades patológicas de fondo como la diabetes mellitus, hipertensión arterial, sobre peso y obesidad $(9,26)$. Especialmente importante en la época actual donde las enfermedades crónico-degenerativas y las expectativas de vida han cambiado drásticamente la funcionalidad sexual femenina, siendo un problema frecuente y complejo, el cual debe de ser estudiado ampliamente para poder tomar medidas pertinentes y prontas para así lograr un manejo y recuperación de la paciente en todos los aspectos biopsicosociales.

\section{CONCLUSIONES}

En el estudio de las pacientes con disfunción sexual, se debe evaluar el estado clínico de la mujer, el emocional y las condiciones sociales al momento de la aparición del problema. El aspecto conyugal debe de ser manejado cuidadosamente para lograr un adecuado acercamiento médicopaciente, para planificar un adecuado manejo. El problema debe ser abordado ampliamente desde un punto de vista de pareja, para orientar y tomar medidas oportunas para lograr la recuperación de la paciente en todos los aspectos biopsicosociales.

\section{REFERENCIAS}

1. Chavarría-Olarte ME, Palomo-Piñón S, Danel I, Granados-Martínez J. La investigación en la salud de la mujer en el siglo XXI. Cir Ciruj 2002;70:194-9. Disponible en: http://www.medigraphic.com/pdfs/circir/ cc-2002/cc023m.pdf.

2. Fonseca H, Greydanus D. Sexuality in the child, teen and young adult: concepts for the clinician. Prim Care 2007;34:275-92.

3. Downey Jl. What women want: psychodynamic of women's sexuality in 2008. J Am Acad Psycoanal Dyn Psychiatry 2009;37:253-68.

4. Gaceta UNAM. El cambio de la identidad femenina según Lipovetsky. Rev Fac Med UNAM 2007;50:1. Disponible en: http://www.medigraphic.com/pdfs/facmed/un-2007/un076k.pdf

5. Goncalves R. Merighi MA. Reflections on sexuality during the climateric. Rev Lat Am Enfermagem 2009;17:160-6.

6. Quijano Narezo M. El origen de la vida: la sexualidad y la muerte. RevFacMed UNAM 2000;43:1-2. Disponible en: http://www.medigraphic.com/pdfs/facmed/ un-2000/un005b.pdf

7. Shorter E. About the history of sexuality. NeuropyschPharmacol Hung 2007;9:31-3.

8. Okeahialam BN, Obeka NC. Sexual dysfunction in female hypertensives. J Natl Med Assoc 2006;98:63840.

9. Shah MB. Obesity and sexuality in women. Obstet Gynecol Clin North Am 2009;36:347-60.

10. Wiltmoth MC. Sexuality a critical component of quality of life in chronic disease. Nurs Clin North Am 2007;42:507-4.

11. Artal R. Women and obesity. Preface. Obstet Gynecol Clin North Am 2009;36:XV-XVI.

12. Kaneshiro B, Jensen JT, Carlson NE, Harvey SM, Nichols MD, Edelman AB. Mass Index and sexual behavior. Obstet Gynecol 2008;112:586-92.

13. Meuris S. Elderly people's sexuality. Rev Med Brux 2007;28:356-8.

14. Nappi RE, Lachowsky M. Menopause and sexuality: prevalence of symptoms and the impact of quality of life. Maturitas 2009;63:138-41.

15. Najjar Abdo $\mathrm{CH}$, Junqueira Fleury $\mathrm{H}$. Diagnostic and therapeutic aspects of female dysfunctions. Rev Psiq Clin 2006;33:162-7.

16. Basson R. Women's sexual dysfunction: revised and expanded definition. CMAJ 2005;172:1327-33.

17. Kammerer-DoaD, Roger RG. Female function and dysfunction. Obstet Gynecol North Am 2008;35:16983.

18. Clayton $\mathrm{AH}$, Hamilton DV. Female sexual dysfunction. Obstet Gynecol North Am 2009;36:861-76.

19. Kingsberg SA, Janata JW. Female sexual disorders: Assesment, diagnosis and treatment. Urol Clin North Am 2007;34:497-506.

20. Laumann EO, Paik A, Rosen RC. Sexual dysfunction in the United States: prevalence and predictors. JAMA 1999;281:537-44.

21. Chávez-Aguilar V, Velasco-Orellana R. Disfunciones 
familiares del subsistema conyugal. Criterios para su evaluación. Rev Med IMSS Méx 1994;32:39-43.

22. Grajales T, Valderrama A. Test de Autoestima. 2000. México, Centro de Investigaciones Educativas Montemorelos.

23. Martínez IJ. Versión Española del cuestionario de Yesavage abreviado: adaptación y validación. MEDIFAM 2002;12:620-30.
24. Smilkstein G. The family APGAR: a proposal for a family function test and its use by physicians. J Fam Pract 1978;6:1231-9.

25. Méndez Castellano H, Méndez MC. Estratificación social y biología humana: método Graffar modificado. Arch Venez Pueric Pediatr 1986;49:93-104.

26. McCall-Hosenfeld J. Sexual satisfaction and cardiovascular disease. The women health iniciative. Am J Med 2008;121:295-301. 